\title{
Spatial approach to analyzing dynamics of racial diversity in large U.S. cities: 1990-2000-2010
}

\author{
Anna Dmowska ${ }^{1}$, Tomasz F. Stepinski ${ }^{1}$ \\ ${ }^{a}$ Institute of Geoecology and Geoinformation, Adam Mickiewicz University, Poznan, Poland \\ ${ }^{b}$ Space Informatics Lab, Department of Geography, University of Cincinnati, Cincinnati, USA, OH 45221-0131, USA
}

\begin{abstract}
Over the last several decades large U.S. cities became increasingly racially diverse. Understanding spatio-temporal dynamics of this significant social change and identifying its broad trends is important for numerous stakeholders. High resolution population grids, which recently become available for the entire conterminous U.S. and for three time points from 1990 to 2010, are an ideal dataset for analyzing dynamics of racial diversity. Their value to diversity analysis has been already demonstrated at the level of the entire U.S. as well as at the level of an individual city. In this paper we demonstrate their value for performing a survey aimed at synthesizing diversity dynamics from different cities in order to identify prevalent nationwide trends. Our survey consists of 41 large cities. 1990-20002010 snapshots of racial condition at each city are provided by respective grids in which each cell is assigned one of nine possible diversity/dominant race categories. All cells with the same diversity label constitute a zone which we refer to as community and measure using a percentage of landscape (PLAND) and an aggregation index (AI) metrics. An inclusion of the AI metric makes possible to determine not only whether a given community grows or shrinks but also whether it's merging or fragmenting. We analyze spatio-temporal evolution of communities by tracking changes in the pairs of the values of these metrics. To simplify we categorize these changes into eight categories resulting in 64 possible change trajectories for each community. Trajectories are histogrammed to reveal variety or scarcity of possible modes of change. Frequent trajectories are identified with broad trends. Eight such trends are identified, they represent most prevalent racial dynamics in the U.S. during the decades of 1990-2010. Two trends correspond to decay of whites-only and blacks-only communities. The remaining six trends correspond to expansion of Hispanics, Asian, and racially diverse communities. Trends do not show regional dependence, they truly reflect profound social change occurring across the entire U.S.
\end{abstract}

Keywords:

Social landscape, race/diversity, landscape metrics, urban change

\section{Introduction}

The Unites States is overall a multiethnic society, but on smaller spatial scales of a single city or an individual neighborhood, it has been and still is quite segregated. However, in the last decades segregated character of American cities and neighborhoods is eroding (Iceland et al., 2002; Iceland, 2004; Farrell and Lee, 2011; Wright et al., 2014; Zhang and Logan, 2016) due to increased immigration from non-European countries and shifting social attitudes. Understanding long-term directions of racial/ethnic character of U.S. cities and

${ }^{*}$ Corresponding author. Email address: stepintz@uc.edu neighborhoods is of great interest to numerous stakeholders including academics, policy makers, and business community. Because of this interest there exist numerous studies devoted to assessing changes in racial diversity at the spatial level of individual cities.

Iceland et al. (2002) and Iceland (2004) measured change over the 1980-2000 period in segregation and diversity of 325 American cities with population larger than 50,000. They used entropy index (Massey and Denton, 1988) to track changes in an overall diversity of a city and the information theory index to track changes in how evenly different racial groups are distributed across city neighborhoods. Farrell (2008) and Farrell and Lee (2011) calculated the same two indices for 100 largest U.S. cities in 1990 and 2000 to test three differ- 
ent hypotheses for a prevailing trend in changing racial structure. Fischer et al. (2004) also used the information theory index in a comprehensive study of diversity change in 175-331 largest metropolitan areas during the 1960-2000 period. Logan et al. (2004) measured change over the 1980-2000 period in segregation between two groups (combinations of whites, blacks, Hispanics, and Asians) in up to 255 cities using the index of dissimilarity (White, 1986; Massey and Denton, 1988).

The aforementioned studies relied on indices calculated from mutually permeating subpopulations of different racial groups to assess change in segregation/diversity, but another research thread used an approach which relies on classification of neighborhoods on the basis of the distribution of residents by race. Logan and Zhang (2010) classified local areas (census tracts) and tracked differences in their classification category between 1980 and 2000. In the follow up study Zhang and Logan (2016) performed a similar analysis of diversity change using a larger sample of 342 cities and a longer time period (1980-2010). Another tract-based classification method was used by Fasenfest et al. (2004) to quantify 1990-2000 change in racial character in 10 largest U.S. cities. Farrell and Lee (2011) classified tracts into 100 major metropolitan areas into diversity/dominant race categories and analyzed change using matrices showing transitions between tracts category assignments during 1990-2000 period. Wright et al. (2014) used similar classification technique applied to 53 major metropolitan areas using data from 1990, 2000, and 2010. The same methodology, but applied to high resolution grid rather than census tracts, was used in Dmowska and Stepinski (2016) to calculated a transition diagram tracing how the entire U.S. population redistributes among different diversity/dominant race categories during the 1990-2000 period.

Although approaches to assessing change in the racial character of U.S. cities vary (see above), their broad conclusions are qualitatively the same: the whitedominated urban neighborhoods are rapidly decaying, predominantly Hispanic and Asian neighborhoods are on the rise, and black-dominated neighborhoods remain stable. However, although a broad direction of change in racial character of American cities is now established, important details on spatio-temporal dynamics of these changes were not addressed by the previous studies. For such details to emerge an explicitly spatial analytic approach needs to be applied to high resolution population data.

Since 2013 we have been working (Dmowska and Stepinski, 2014, 2016, 2017; Dmowska et al., 2017) on making such approach possible. The result of this work is an interactive GeoWeb application SocScape and an associated database (both available at http://sil.uc.edu) which display/provide $30 \mathrm{~m} /$ cell grids of U.S. population, its subpopulations, as well as its classification into diversity/dominant race categories. The grids are the results of dasymetric modeling of 1990, 2000, and 2010 census block-level data. Details of racial dynamics can best be observed using change maps constructed from the grids for 363 major metropolitan areas (Dmowska et al., 2017). Examples of such maps for Chicago, Houston, and San Francisco can be seen in Dmowska and Stepinski (2016). They can also be observed for any place in the conterminous U.S. using SocScape for sideby-side comparison of diversity/dominant race maps at 1990, 2000, and 2010, example of such comparison for the north-eastern part of Atlanta, GA is shown in Fig. 1.

In this paper we provide a synthesis of racial dynamics in selected large U.S. cities based on high resolution diversity/dominant race grids (hereafter referred to as diversity grids for short). Because we use gridded data instead of areal units (like tracts) our approach to the synthesis is significantly different from those used in previous studies. We track time evolution (hereafter referred to as a trajectory) of different "communities" in each city in our survey. In this paper a community means a zone comprising all locations (cells in a grid) assigned to the same diversity category. It also mean a specific mix of different racial group as indicated by a category label; a presumption is that such mix correlates with a broader social character since the name community.

For a given community we gather their change trajectories from all cities in our survey and categorize them to reduce complexity of the data. A histogram of categorized trajectories shows a range of dynamics this particular community experienced in different U.S. cities. A prominent peak in the histogram indicates an existence of a trend - a specific dynamics that occurred in a significant fraction of the cities in the survey. We search for such trends among all communities. The end result of our synthesis is a list of all identified trends. These trends highlight most widespread patterns of racial change in large U.S. cities during the 1990-2010 period. We also look for possible regional differences in trends as well as less frequent change trajectories.

\section{Data and methods}

SocScape $30 \mathrm{~m} /$ cell resolution racial diversity grids for years 1990, 2000, and 2010 (Dmowska et al., 2017) 

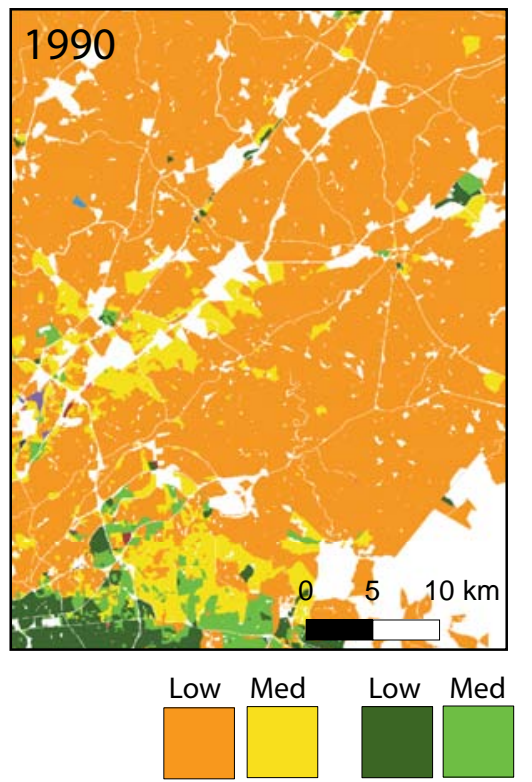

White

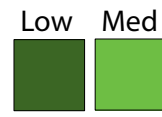

Black

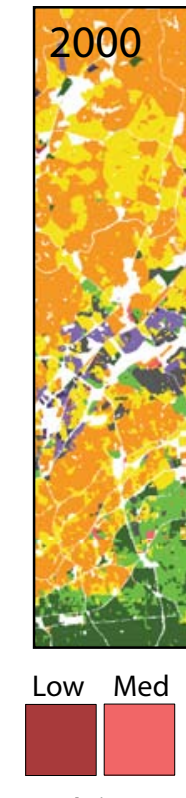

Asian
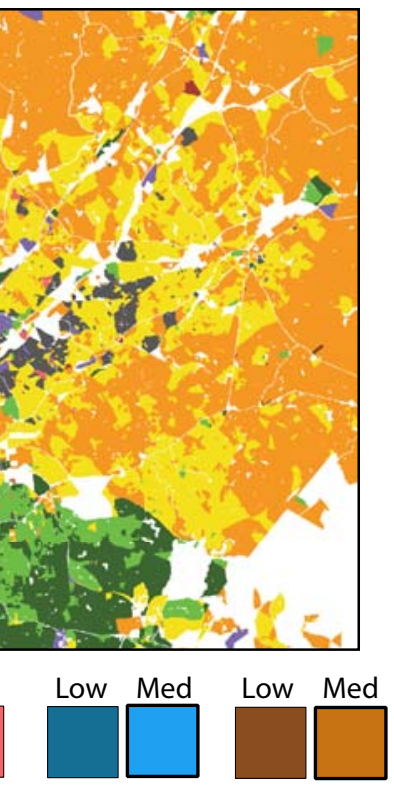

Am.Indian
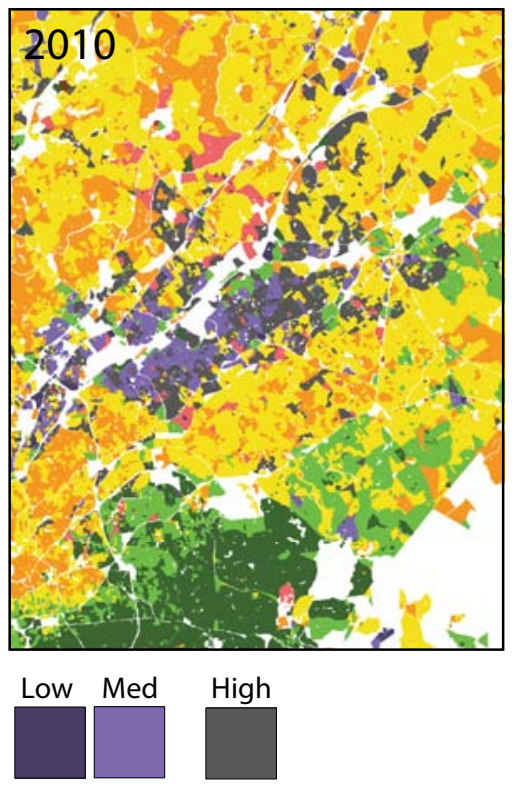

Hispanic

Figure 1: Racial diversity grids-based maps of the north-eastern part of Atlanta, GA in 1990, 2000, and 2010. The legend of communities refer to the level of racial diversity (top labels) and dominant race (bottom label).

constitute an input data for our investigation. From this dataset we extracted grids for a selection of 41 cities defined by the extent of urban areas within their MSA. Our criteria for the selection were as follows: (1) the population of the entire MSA was over 1 million in 2010, and (2) all nine diversity/dominant race communities (listed below) were present in 1990, 2000, and 2010. These grids are a product of three-dimensional classification of cells into 40 categories (including one indicating uninhabited area) based on criteria of racial diversity, dominant race, and population density (Dmowska and Stepinski, 2014; Dmowska et al., 2017). For the present study we have collapsed the original classification to just 13 classes by eliminating dependence on population density. These classes describe specific racial mixes or communities (see the Introduction section) by commenting on the level of diversity and on the dominant race (if any).

We focus on nine predominant communities: white low diversity (WL), white medium diversity (WM), black low diversity (BL), black medium diversity (BM), Hispanics low diversity (HL), Hispanics medium diversity (HM), Asians low diversity (AL), Asians medium diversity (AM), and high diversity (Hdiv). For example, the white low diversity community (WL) is a zone where white population represents over $80 \%$ of total population in each cell, and the white medium diver- sity community (WM) is a zone where whites are still a majority group but they don't exceed $80 \%$ of cell population. The Hdiv is a zone characterized by the lack of dominant race.

To illustrate how diversity grids depict change in the racial character of a city Fig. 1 shows grid-based maps restricted to a north-eastern part of Atlanta, GA. The progression of maps from 1990 to 2010 clearly shows the WL community being replaced by the WM community over time. Fig. 1 also shows the growth of HM and Hdiv communities from what use to be WM-dominated part of the city in 1990, and an expansion of BM and $\mathrm{BL}$ communities in the north-eastern direction.

\subsection{Measuring community zones}

A site-by-site visual assessment of a progression of diversity maps for a single city (like in Fig. 1) reveals changes in city's racial character in details, however, the results of such assessment performed for multiple cities would be difficult to synthesize. To perform synthesis of racial dynamics over multiple cities we introduce two spatial metrics that summarize magnitude and spatial distribution of each community zone. These metrics could be compared from year to year to give a quantitative measure of the change.

In Dmowska et al. (2017) we noted that diversity maps, like the ones in Fig. 1, have the same data for- 
mat as landscapes in the field of landscape ecology, with the only difference being the actual meaning of map's categories. Thus, if we would to think about diversity maps as "social landscapes" we can analyze them using metrics already developed and implemented in software (McGarigal, 2014; VanDerWal et al., 2014) in the context of ecology. Several such metrics were suggested by Dmowska et al. (2017), here we utilize two of them, percentage of landscape (PLAND) and aggregation in$\operatorname{dex}(\mathrm{AI})$.

PLAND (having a possible range of $0 \%$ to $100 \%$ ) refers to percentage of a city's area inhabited by a given community. Assuming, that number of people in a given community is proportional to zone's area, PLAND measures a magnitude or a degree of contribution of this community to the city. Changes in PLAND indicate growing or shrinking of a community. For example, in the region depicted in Fig. 1, PLAND $=74.2 \%$ for the WL community in 1990 , but PLAND value drops to just $15.3 \%$ in 2010 . Clearly, the WL community has shrank during this period. Descriptively, whereas in 1990 an observer randomly placed in this region would have $74.2 \%$ chance of finding himself in a white-only neighborhood, his chance of doing so in 2010 would be only $15.3 \%$. At the same time the WM community has grown from $8.4 \%$ to $38.8 \%$.

AI (having a theoretical range of 0 to 100) describes the level of spatial aggregation of a zone associated with a given community. $\mathrm{AI}=0$ means that the community zone is maximally spatially disaggregated (there are no like adjacencies between cells comprising the zone). $\mathrm{AI}=100$ means that community zone is maximally aggregated into a single compact clump. The nature of diversity communities is such that small values of AI are not occurring so the actual numerical range of AI is narrow and concentrated at large values. For example, in the region depicted in Figs. 1 and 3, the 1990 WL community zone is the most aggregated $(\mathrm{AI}=98.1)$ whereas 1990 and 2000 AM community zones are the least aggregated ( $\mathrm{AI}=84.7$ and 87.7 , respectively). Changes in AI measure merging or fragmenting of a community zone - an aspect of population dynamics that is of significant importance for an overall change in the racial character of a city.

We used SDMTools - Species Distribution Modeling Tools (VanDerWal et al., 2014) for R software to calculate 1107 (41 cities $\times 9$ communities $\times 3$ time instances) pairs of (PLAND,AI) values.

\subsection{Calculating change trajectories}

Given three observation times (1990, 2000, and 2010) there are two periods of change, 1990-2000 and 2000-

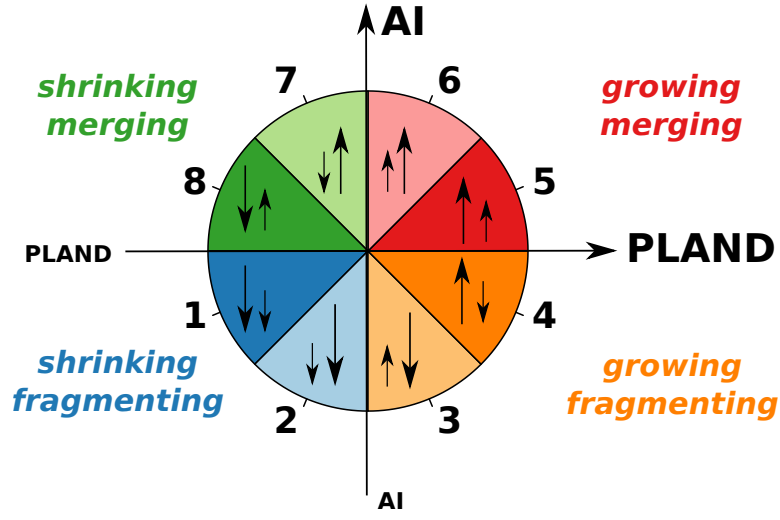

Figure 2: Eight categories of change. Left arrow indicates change of PLAND and right arrow indicates change of AI. Length of arrow indicates which index changed more.

2010. For a particular community in a given city we first calculate relative change of PLAND and AI for each change period separately,

$$
\delta_{i+10, i}^{X}=\left(X_{i+10}-X_{i}\right) / X_{i}
$$

where $X$ refers to either PLAND or AI, $i=1990$ or 2000 , and $\delta_{i+10, i}^{X}$ is the change during 10 years relative to the value of an index in the earlier year.

To simplify the change data we classify the pairs $\left(\delta^{P L A N D}, \delta^{A I}\right)$ for a given change period into eight categories, labeled by numbers from 1 to 8 as shown in Fig.2. This reduces 1107 unique pairs of numbers into 1107 non unique pairs of labels facilitating a synthesis of the results. The four quarters (depicted by green, blue, orange, and red colors) correspond to four major types of change. Each quarter is further divided into two sub-sections (depicted by different shades of a color) to indicate which of the two main modes of change (growing/shrinking and merging/fragmenting) dominates. For example, change categories 5 and 6 both correspond to growing and merging, but in category 5 growing is a dominant process of change, whereas in category 6 merging is a dominant process. With such a categorization a community change can be referred to by a single number form 1 to 8 .

A change trajectory for a particular community in a given city is a pair of change categories where the first element of the pair indicates change during the 19902000 period and the second element indicates change during the 2000-2010 period. For example, the black low diversity (BL) community in Chicago is assigned trajectory $(4,2)$ meaning that in the decade of 1990 2000 it grew but also fragmented although the rate of fragmenting was lower than the rate of growing, but in 

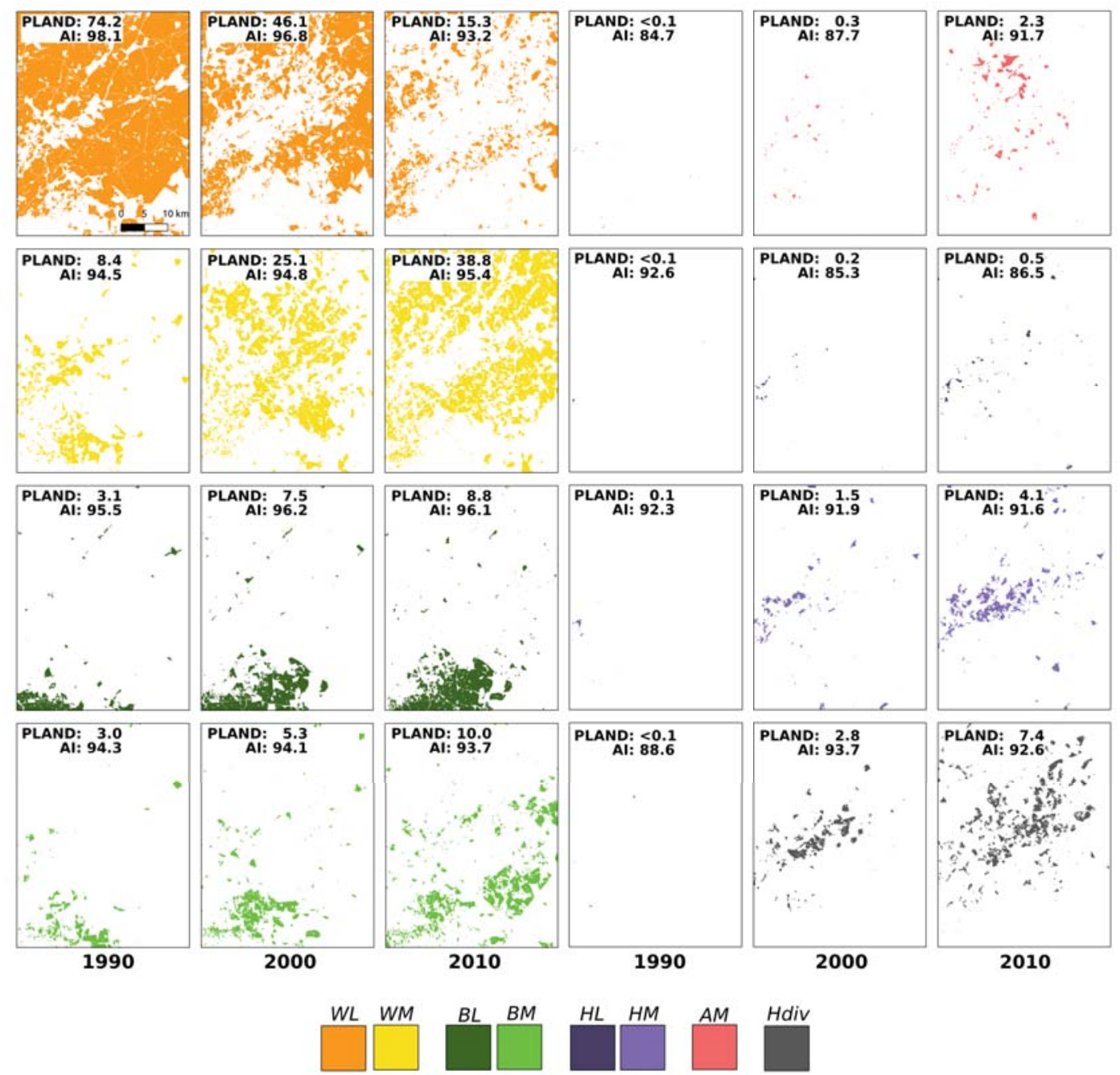

Figure 3: Spatial distribution of eight (out of nine) community zones in the north-eastern part of Atlanta, GA in 1990, 2000 , and 2010.

the decade of 2000-2010 it reversed its growth, started shrinking while still fragmenting and the rate of shrinking was lower than the rate of fragmenting. On the other hand the Asian medium diversity (AM) community in San Francisco is assigned trajectory $(4,5)$ meaning that in the decade of 1990-2000 it grew but also fragmented, but in the decade of 2000-2010 it continue to grow and started merging.

Because there are 8 possible changes in each period, there are 64 theoretically possible change trajectories for each community. A dominant trend of change for a given community is a trajectory identified in a significant fraction of cities in our survey. For example, for the Hispanics medium diversity (HM) community we have found change trajectory $(5,5)$ in 19 out of 41 cities in our survey (46\%). This is a high enough percentage to constitute a trend. It makes possible the following statement: in about half cities in our survey the communities with dominant Latino populations grew and spatially consolidated during 1990s and 2000s.

\section{Results}

In the first step we decomposed 1990, 2000, and 2010 diversity maps for each city in our survey into nine separate maps each showing spatial extent of specific community zone and calculated their change trajectories. Fig. 3 shows this decomposition for a region in the north-eastern part of Atlanta (a subset of Atlanta MSA). 

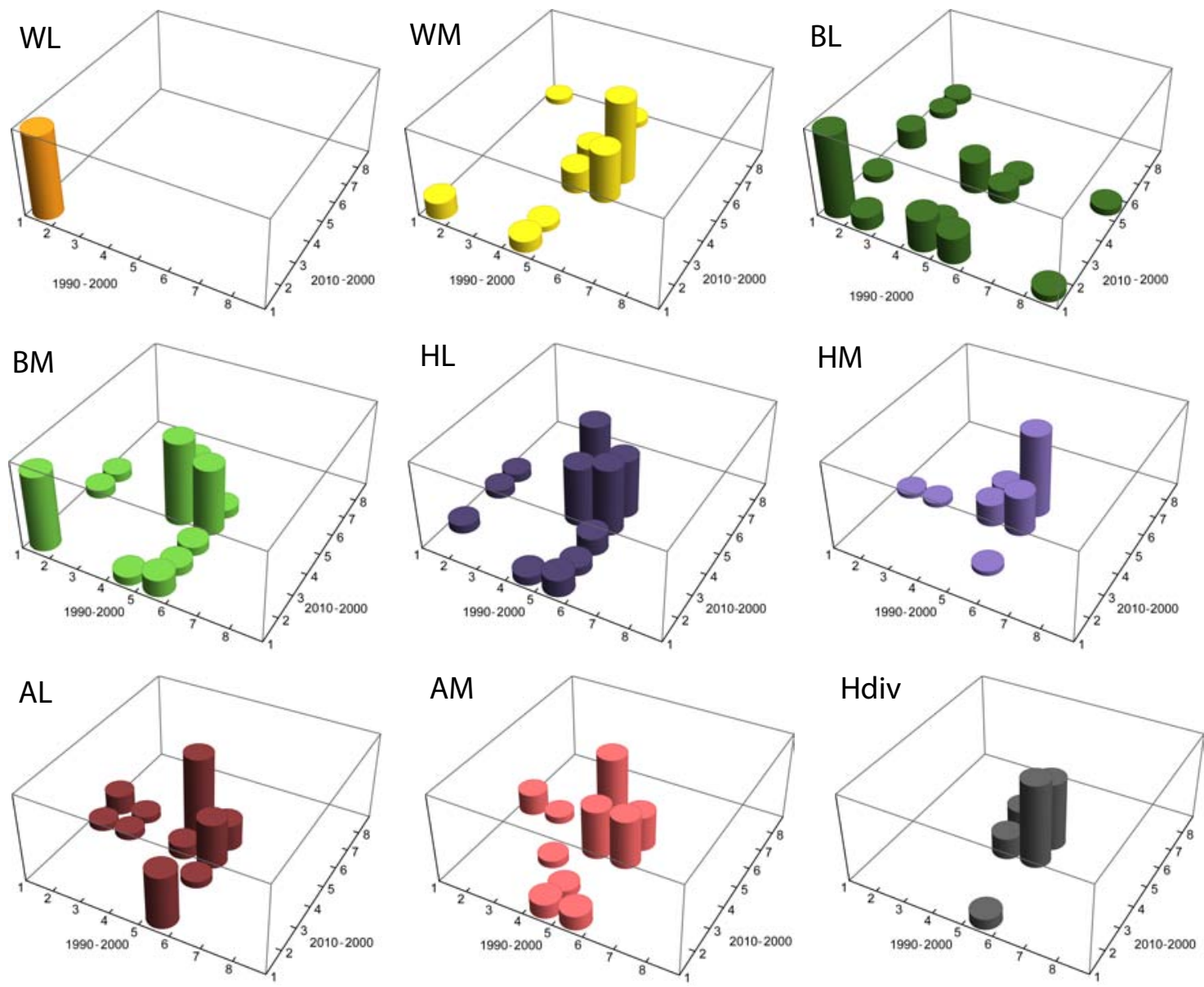

Figure 4: Histograms of change trajectories for each of the nine communities. Bin heights indicate the number of cities in our survey which followed a given change trajectory during 1990-2000-2010. The x-axis indicates 1990-2000 change label and the y-axis indicates $2000-2010$ change labels. For meaning of change labels see Fig. 2.

Only eight (out of nine) community zones are shown because the AL zone was too small (PLAND $<0.1 \%$ ) to be noticeable. This figure has eight sections (one for each community zone) and each section has three panels, one for each year. Values of PLAND and AI are given in the top left corner in each panel, change trajectories are calculated from these values.

This example illustrates five different change trajectories. The WL community evolved along the $(1,1)$ trajectory - it shrank and fragmented in both decades. This represents a decline of whites-only neighborhoods. The WM and AM communities evolved along the $(5,5)$ trajectory - they grew and merged in both decades. In the case of WM it represents an erosion of WL by steady influx of people of different communities into what previously were white only neighborhoods. In the case of AM it represents addition and enlargement of Asian- dominated neighborhoods. The BM and HM communities evolved along the $(4,4)$ trajectories - they grew but fragmented in both decades. The fragmenting is relative, it does not necessarily means that established enclaves of BM and HM communities split, but rather that in a later period their entire (enlarged) zones had more patches which is one of possible modes of expansion. The BL and Hdiv communities along the $(5,4)$ trajectory - they grew and merge in the 1990s but grew and fragmented in 2000s. This illustrates two different modes of growing, first by expanding around already established core locations, then by establishing new core locations. Finally, the HL community evolved along the $(4,5)$ trajectory which is a reverse of the $(5,4)$ - this community grew in the 1990 s by establishing new core locations, and it grew in 2000 s by enlarging already established locations. 
Table 1: Trends of racial composition change in 1990-2000-2010

\begin{tabular}{|c|c|c|c|c|c|}
\hline Rank & Community & Trajectory & \# of cities $(\%)$ & Description & Cities \\
\hline 1 & WL & $(1,1)$ & $41(100)$ & $\begin{array}{l}\text { Shrinking/fragmenting } \\
\text { both decades }\end{array}$ & All 41 cities \\
\hline 2 & HM & $(5,5)$ & $19(46)$ & $\begin{array}{l}\text { Growing/merging } \\
\text { both decades }\end{array}$ & $\begin{array}{l}\text { San Antonio, Tucson, Dallas, San Francisco, New } \\
\text { York, Tampa, Las Vegas, Orlando, Providence, Ok- } \\
\text { lahoma City, Kansas City, New Orleans, Portland, } \\
\text { Cleveland, Harrisburg, Minneapolis, Jacksonville, } \\
\text { Greenville, Virginia Beach }\end{array}$ \\
\hline 3 & Hdiv & $(5,4)$ & $16(39)$ & $\begin{array}{l}\text { Growing/merging }+ \\
\text { Growing/fragmenting }\end{array}$ & $\begin{array}{l}\text { San Francisco, Sacramento, Los Angeles, Houston, } \\
\text { Seattle, New York, Albuquerque, Dallas, Phoenix, } \\
\text { Boston, Atlanta, Austin, San Antonio, Virginia } \\
\text { Beach, Orlando, Harrisburg }\end{array}$ \\
\hline 4 & WM & $(5,5)$ & $15(37)$ & $\begin{array}{l}\text { Growing/merging } \\
\text { both decades }\end{array}$ & $\begin{array}{l}\text { Tucson, Miami, Oklahoma City, Denver, Tulsa, New } \\
\text { York, Atlanta, Hartford, Greenville, Kansas City, } \\
\text { Portland, Detroit, Cleveland, Boston, Minneapolis }\end{array}$ \\
\hline 5 & AL & $(4,5)$ & $13(32)$ & $\begin{array}{l}\text { Growing/fragmenting } \\
\text { + Growing/merging }\end{array}$ & $\begin{array}{l}\text { Sacramento, Riverside, Seattle, San Diego, Portland, } \\
\text { Hartford, Orlando, Las Vegas, Tulsa, Tucson, Austin, } \\
\text { Albuquerque, Kansas City }\end{array}$ \\
\hline 6 & BL & $(1,1)$ & $13(32)$ & $\begin{array}{l}\text { Shrinking/fragmenting } \\
\text { both decades }\end{array}$ & $\begin{array}{l}\text { Virginia Beach, Houston, Dallas, Tulsa, Philadelphia, } \\
\text { New York, San Francisco, Los Angeles, Austin, Las } \\
\text { Vegas, Riverside, Boston, Tucson }\end{array}$ \\
\hline 7 & Hdiv & $(5,5)$ & $13(32)$ & $\begin{array}{l}\text { Growing/merging } \\
\text { both decades }\end{array}$ & $\begin{array}{l}\text { Washington DC, Providence, Minneapolis, Denver, } \\
\text { Las Vegas, Miami, Tucson, Hartford, New Orleans, } \\
\text { Portland, Philadelphia, Buffalo, Tampa }\end{array}$ \\
\hline 8 & $\mathrm{AM}$ & $(4,5)$ & $12(30)$ & $\begin{array}{l}\text { Growing/fragmenting } \\
+ \text { Growing/merging }\end{array}$ & $\begin{array}{l}\text { San Francisco, Seattle, Dallas, Chicago, Portland, } \\
\text { Austin, Boston, Atlanta, Virginia Beach, Las Vegas, } \\
\text { Cleveland, Buffalo }\end{array}$ \\
\hline
\end{tabular}

Table S1 in the supplement lists change trajectories for all 369 communities in the entire survey of 41 cities. Of the 64 theoretically possible types of trajectories only 23 types have been identified in our survey: six growing types accounting for 237 trajectories, six shrinking types accounting for 73 trajectories, and 11 mixed types accounting for 59 trajectories. The most popular growth trajectories (regardless of the community they apply to) are $(5,4),(5,5)$, and $(4,5)$, having counts of 66, 65, and 57 trajectories, respectively. The most popular shrinkage trajectory is $(1,1)$ having count of 66 , and the most popular mixed trajectory is $(5,1)$ having count of 20 .

\subsection{Trends}

In the second step we identified dominant trends of racial diversity dynamics in the largest U.S. cities during the period of 1990-2010. Instances of change trajectories for given community are collected from all cities in a survey and histogramed. This is repeated for all nine communities resulting in nine different histograms shown in Fig. 4. These histograms have a 2D form because labels of change trajectories are compound, they consist of a change label for the 1990-2000 period followed by a change label for the 2000-2010 period. The $\mathrm{x}$-axis in histograms indicates the 1990-2000 change label and the y-axis indicates the 2000-2010 label; 41 change trajectories are allocated to 64 possible bins. Prominent peaks in those histograms indicate frequent change trajectories referred to as trends. We set a threshold $\geq 30 \%$ ( 12 or more out of possible 41 ) trajectories in a peak to constitute a trend. Thus, a trend is a character of change a given community underwent during the 1990-2000-2010 period in at least 12 cities in our survey. Given this criterion we identified eight trends; they are listed and summarized in Table 1.

Peaks located in the middle of the histogram correspond to persistent growth of communities over both decades. Histograms for most communities had such peaks, some of them high enough to constitute trends, exceptions are the WL community who has no peaks in this region of the histogram and the BL community who has only very small peaks in this region. Peaks located in the corners of the histogram correspond to persistent shrinking of communities over both decades. Only WL, BL, and BM communities have significant 
peaks in those regions of the histogram and only two of these peaks (for WL and BL) are high enoughs to be considered trends. Peaks located elsewhere in the histogram correspond to mixed trajectories - combination of growth or shrinkage in the first decade followed by the opposite in the next decade. The BL, HL, and AL communities had some peaks in those regions but none high enough to constitute a trend.

The number of different peaks in a histogram signals a large variation in change trajectories for a given community across the cities in the survey. The BL community has the largest number of different trajectories (13) but only one of them (trajectory $(1,1)$ indicating shrinking and fragmenting over the entire two decades) is numerous enough (13 cities) to constitute a trend. The trajectories for the remaining 41-13=28 cities are spread between many different types. This suggests that, apart from the single trend, the dynamics of BL community depends on local circumstances rather than on nationwide tendencies. On the other extreme is the WL community which follows the same trajectory in all cities in the survey. This suggests that WL dynamics is the result of broader, nationwide tendencies.

Trends listed in Table 1 are likely to reflect broader tendencies. Three trends $((5,5),(5,4)$, and $(4,5))$ pertain to a tendency of a community to grow during both decades. Two growing trends are identified for the Hdiv community. The slightly stronger (16 cities) trend $(5,4)$ has been observed predominantly in the West Cost and Southwest cities where Hdiv zones grew around its core areas in the first decade then grew by forming new cores in the second decade. The slightly weaker (13 cities) trend has been observed predominantly in the East Cost and Midwest cities where Hdiv zones grew around its core areas for the entire 1990-2010 period. A $(4,5)$ trend has been identified for the Asian communities (AL and $\mathrm{AM}$ ), these communities grew by forming new cores in the first decade, then increased these cores in the second decade. A $(5,5)$ trend has been identified for HM and WM communities. These communities grew around existing cores for the entire period. The growth of WM is a direct result of the shrinking of WL. We identified a singe $(1,1)$ trend which pertains to a tendency of a community to shrink. It applies to WL and BL communities and it indicates shrinking and fragmenting during the entire period; in case of WL this trend affected all cities in our survey. There are no trends pertaining to mixed growth/shrinkage tendencies.

\subsection{Spatial distribution of change trajectory types}

In the last step we mapped change trajectory types over the entire conterminous U.S. to check for possible regional patterns. Fig. 5 has eight panels corresponding to maps for eight (out of nine) communities, the map for the WL community is not shown because all 41 cities in the survey have the same trajectory $(1,1)$. The cities are marked by symbols encoding trajectory type. The symbol is a two color circle with the color in the center of the circle encoding change type in the 1990-2000 period and the color on the outside of the circle encoding change type in the $2000-2010$ period. Recall that warm colors (change types $3,4,5,6$ ) pertain to growth of communities whereas the cold colors (change types $1,2,7,8)$ pertain to shrinkage of communities. Symbols with black outline indicate rare trajectories, for a given community only a single city has this type of trajectory.

No strong geographical differences in trajectory types are observed for all communities with the exception of the BM community. This community experiences shrinkage in the cities located on the West Cost but it experiences growth in the remaining part of the country. The AL community trend trajectory $(4,5)$ characterizes 13 cities located across the U.S. The second most frequent trajectory for this community $(5,1)$ characterize 8 cities also evenly distributed across the U.S. The same lack of regional differences is observed for the AM, HL, $\mathrm{HM}$, and Hdiv communities. A small geographical difference in change trajectories is observed for the WM community, cities in the West Cost experienced slow growth or shrinkage of this community whereas cities in the rest of the country experiences significant growth of this community.

There are some unique trajectories (those indicated by the black outlines) that need individual attention. An example is the $(1,2)$ trajectory for the HL community in Phoenix, AZ. This trajectory indicates that Hispanicsonly community in Phoenix was shrinking during the entire period of 1990-2010, even so Hispanic population in the entire city grew significantly during this period. This indicates a strong integration of Hispanics with whites as evident from growing HM and WM communities. Another example is the trajectory $(1,3)$ for El Paso, TX which indicates shrinkage in the first decade followed by growth in the second decade. As population of El Paso is dominated by Hispanics, this trajectory reflects interchange between HL and HM communities. Relatively to its entire population, sections of El Paso where Hispanics were integrated with whites (while still being a majority) shrank during the $1990-2000$ period in favor of Hispanics-only sections, while it rebounded in the 2000-2010 period. Note that in the 2000-2010 period the HL community also grew, indicating overall Hispanicization of El Paso. 


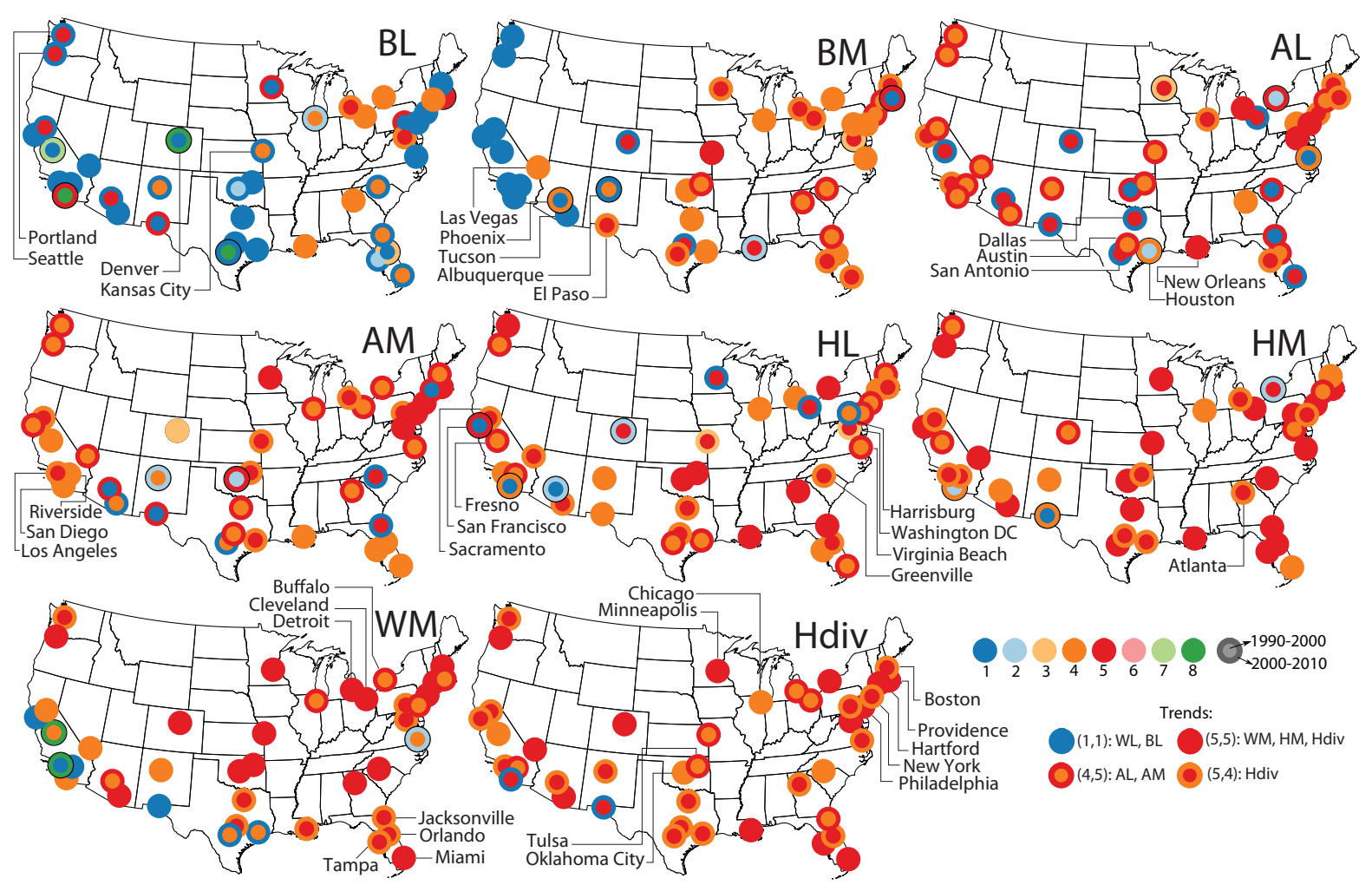

Figure 5: Spatial distribution of change trajectories. Panels correspond to communities as labeled. Cities in the survey are indicated by labels encoding a particular change trajectory. See Fig. 2 for interpretation of change labels.

\section{Discussion and Conclusions}

We have previously argued (Dmowska et al., 2017) for using newly available high resolution population grids instead of census aggregated areas to get more insight into racial dynamics in the U.S. In Dmowska and Stepinski (2016) we demonstrated how grids can be used to construct a detailed change diagram summarizing racial dynamics on the scale of the entire country. In the same paper we also demonstrated how they can be used to obtain detailed maps of diversity change on the scale of an individual city. In the present paper we completed our argument by demonstrating utility of population grids for multi-city survey of racial dynamics. Taken together, these arguments and demonstrations show that grid-based analysis reveals more insights and details regarding this important social phenomenon than previous methodology based on aggregated data. Some of these advantages follows from greater resolution of gridded data, while most follows from greater suitability of grid data format for quantitative spatial analysis.
Our analysis uses several non-standard notions. The most important notion is that of dividing a city into nonoverlapping zones each corresponding to a specific diversity/dominant race condition (which we call communities for short). Definitions of the zones remain fixed over time, thus mapping the zones in successive time points visually reveals racial dynamics in spatiotemporal fashion. A notion of dividing a city into zones on the basis of diversity/dominant race has been used before (Logan and Zhang, 2010; Logan et al., 2015; Holloway et al., 2012; Wright et al., 2014) but the zones were spatially coarse as they consists of census tracts, and temporal analysis was made difficult by the fact that boundaries of tracts changed between time points.

The second important notion is the spatial approach to analysis. Sub-populations of different racial/ethnic groups are counted to produce diversity grid, but our analysis, which uses diversity grid as input data, rely only on spatial relations. Communities are considered spatial zones and the two metrics we use to measure them both pertain to their spatial properties. In the case of PLAND metric it is possible to use population per- 
centage instead of an area percentage, but we decided to keep the original definition of PLAND because changing area to population has negligible impact on conclusions.

The third novelty is to use change trajectories instead of year-to-year comparison. Granted, our trajectories consist of only three time points, but comparing cities on the basis of even such short trajectories give more information than comparing them on year-to-year basis. Previously, only Bader and Warkentien (2016) used trajectories to asses racial dynamics, but their analysis was restricted to four cities (New York, Los Angeles, Chicago, and Houston) and their trajectories were calculated for each tract separately.

Fig. 4 represents a compact summarization of our results. For each community it shows a histogram of change trajectories followed by these communities in the cities in our survey. An overall character of a histogram reveals spectrum of possible change trajectories for a given community. A very compact histogram, like the one for the WL community, indicates broad scale social phenomenon steering dynamics of the community. In the case of the WL community it indicates its decline over the last two decades everywhere in the U.S. as documented by all previous studies listed in the Introduction section. Histogram having many non-empty, approximately equal height bins would indicate absence of broad scale social phenomenon, instead all changes would likely be due to local circumstances. As none of the nine histograms in Fig. 4 has such character, changes to all communities are likely to be steered in large part by broad scale social forces.

Some change trajectories are especially frequent, we refer to them as trends. We identified eight trends and listed them in Table 1, they represent most prevalent racial dynamics in the U.S. during the decades of 19902010. Thus, Table 1 can be considered as a bottom line conclusion of our study, these eight trends describe most important aspects of diversity change in the U.S. cities during 1990-2000 in a compact and lucid manner. Identified trends are broadly in agreement with those found in the previous studies. However, because of our use of spatial analysis and because we use trajectories instead of year-to-year comparisons, some additional information is available even from the summary in Table 1 (column 3). Usage of aggregation index as a metric provides an additional information beyond growing/shrinking. This information pertains to changing topology of community zone and we refer to it as merging/fragmenting. It is easy to see why such information is important, for example, it can distinguish between different modes of community growth. In the first mode (growth + merge) community expands from a priori established enclaves but in the second mode (growth + fragmenting) it grows by establishing new enclaves. Such insight can be helpful to understanding an overall process of changing racial/diversity character of U.S. cities.

With gridded data for the entire conterminous U.S. now publicly available from http://sil.uc.edu similar surveys could be performed for location other than large cities, such as rural areas, small cities, or suburbia. Also, whereas we use only two landscape metrics in our survey, more metrics suitable for description of social landscapes are listed and discussed in Dmowska et al. (2017). Finally, once 2020 Census data will be published, we will calculate a 2020 grid and change trajectories would be extended from three to four time points.

\section{References}

Bader, M. D., Warkentien, S., 2016. The fragmented evolution of racial integration since the Civil Rights Movements. Sociological Science 3, 133-166.

Dmowska, A., Stepinski, T. F., 2014. High resolution dasymetric model of U.S. demographics with application to spatial distribution of racial diversity. Applied Geography 53, 417-426.

Dmowska, A., Stepinski, T. F., 2016. Mapping changes in spatial patterns of racial diversity across the entire United States with application to a 1990-2000 period. Applied Geography 68, 1-8.

Dmowska, A., Stepinski, T. F., 2017. A high resolution population grid for the conterminous United States: The 2010 edition. Computers, Environment and Urban Systems 61, 151-161.

Dmowska, A., Stepinski, T. F., Netzel, P., 2017. Comprehensive framework for visualizing and analyzing spatio-temporal dynamics of racial diversity in the entire United States. PLoS ONE 12(3), e0174993.

Farrell, C. R., 2008. Bifurcation, fragmentation or integration? The racial and geographical structure of US metropolitan segregation, 1990-2000. Urban Studies 45, 467-499.

Farrell, C. R., Lee, B. A., 2011. Racial diversity and change in metropolitan neighborhoods. Social Science Research 40 (4), 1108-1123.

Fasenfest, D., Booza, J., Metzger, K., 2004. Living Together: A new look at racial and ethnic integration in metropolitan neighborhoods, 1990-2000. Tech. rep.

Fischer, C. S., Stockmayer, G., Stiles, J., Hout, M., 2004. Distinguishing the geographic levels and social dimensions of US metropolitan segregation, 1960-2000. Demography 41(1), 37-59.

Holloway, S. R., Wright, R., Ellis, M., 2012. The racially fragmented city? Neighborhood racial segregation and diversity jointly considered. The Professional Geographer 64, 63-82.

Iceland, J., 2004. Beyond Black and White metropolitan residential segregation in multi-ethnic America. Social Science Research 33, 248-271.

Iceland, J., Weinberg, D., Steinmetz, E., 2002. Racial and ethnic residential segregation in the United States: 1980-2000. Tech. rep.

Logan, J. R., Stults, B. J., Farley, R., Stults, J., 2004. Segregation of minorities in the metropolis: Two decades of change. Demography 41 (1), 1-22.

Logan, J. R., Zhang, C., 2010. Global neighborhoods: New pathways to diversity and separation. American Journal of Sociology 115 (4), 1069-1109. 
Logan, J. R., Zhang, W., Turner, R., Shertzer, A., 2015. Creating the Black ghetto: Black residential patterns before and during the Great Migration. The ANNALS of the American Academy of Political and Social Science 660 (1), 18-35.

Massey, D. S., Denton, N. A., 1988. The dimensions of residential segregation. Social Forces 67(2), 281-315.

McGarigal, K., 2014. FRAGSTATS Help. Documentation for FRAGSTATS 4.0. University of Massachusetts, Amherst.

VanDerWal, J., Falconi, L., Januchowski, S., Shoo, L., Storlie, C., 2014. SDMTools: Species Distribution Modelling Tools: Tools for processing data associated with species distribution modelling exercises. R package version 1.1-221.

URL https : //CRAN . R-project .org/package=SDMTools

White, M. J., 1986. Segregation and diversity measures in population distribution. Population Index 52(2), 198-221.

Wright, R., Ellis, M., Holloway, S. R., Wong, S., 2014. Patterns of racial diversity and segregation in the United States: 1990-2010. The Professional Geographer 66(2), 173-182.

Zhang, W., Logan, J. R., 2016. Global neighborhoods: Beyond the multiethnic metropolis. Demography 53 (6), 1933-1953. 